\title{
National Service Center, St. Louis County, Missouri - USA
}

\section{emplazamiento}

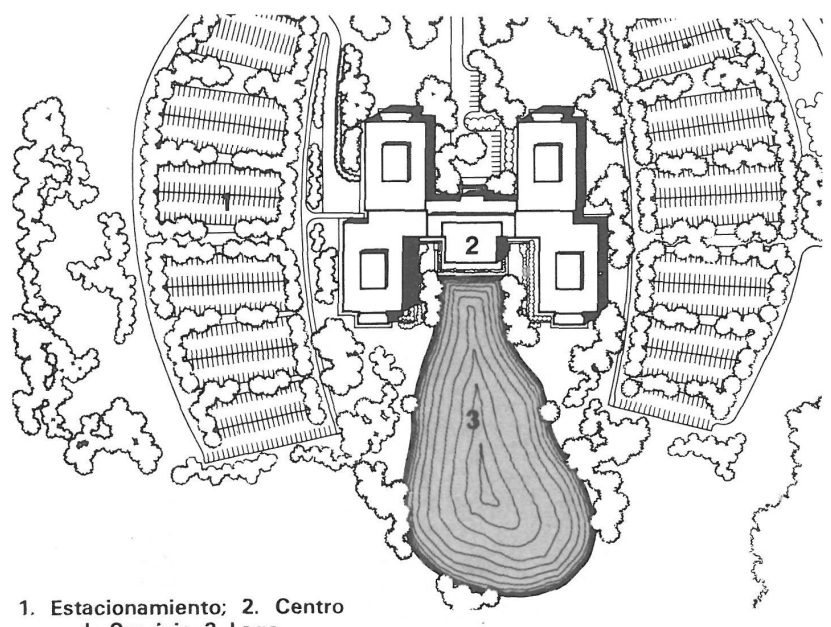

Hellmuth, Obata y Kassabaum, arquitectos

$145-103$

\section{sinopsis}

La nueva sede del National Service Center, situada en una parcela de mas de $40 \mathrm{Ha}$ en el extrarradio de la ciudad, parece un campus universitario más que un centro administrativo. El edificio, que consta de sólo dos plantas sobre rasante, procura su integración en un paisaje marcadamente rural evitando la posible imposición agresiva de la arquitectura en el entorno natural. Con este fin se emplearon colores terrosos, en el acabado de los paneles prefabricados de cemento, complementados por una carpinteria de marcado trazado horizontal en tonos bronce oscuro.

Interiormente el edificio destaca por su flexibilidad, constituyendo espacios de oficinas abiertos e inteconstos. Su estructura modular permite la grados. Su estructura modular permite la ampliación sión actual, sin alterar la unidad arquitectónica.

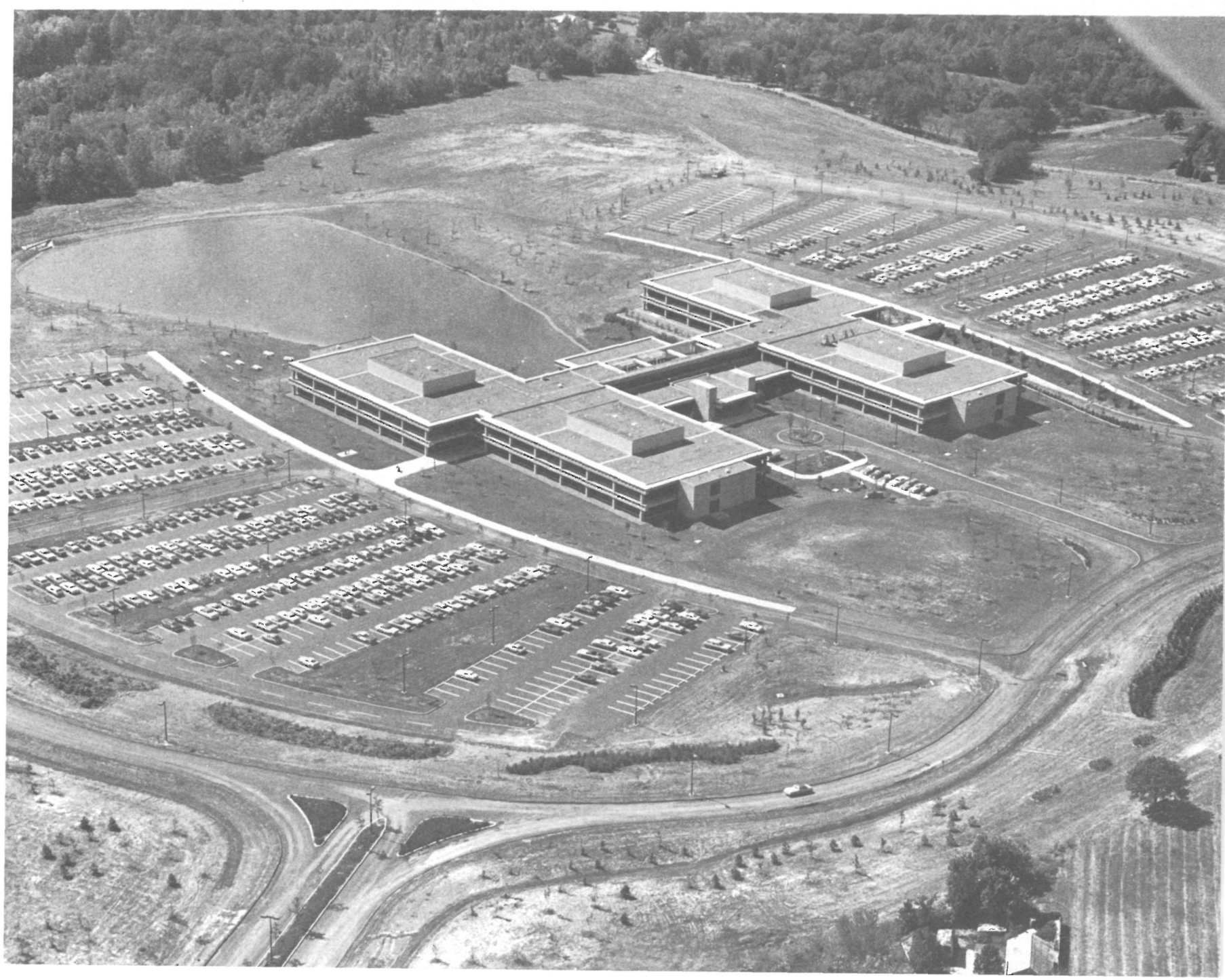



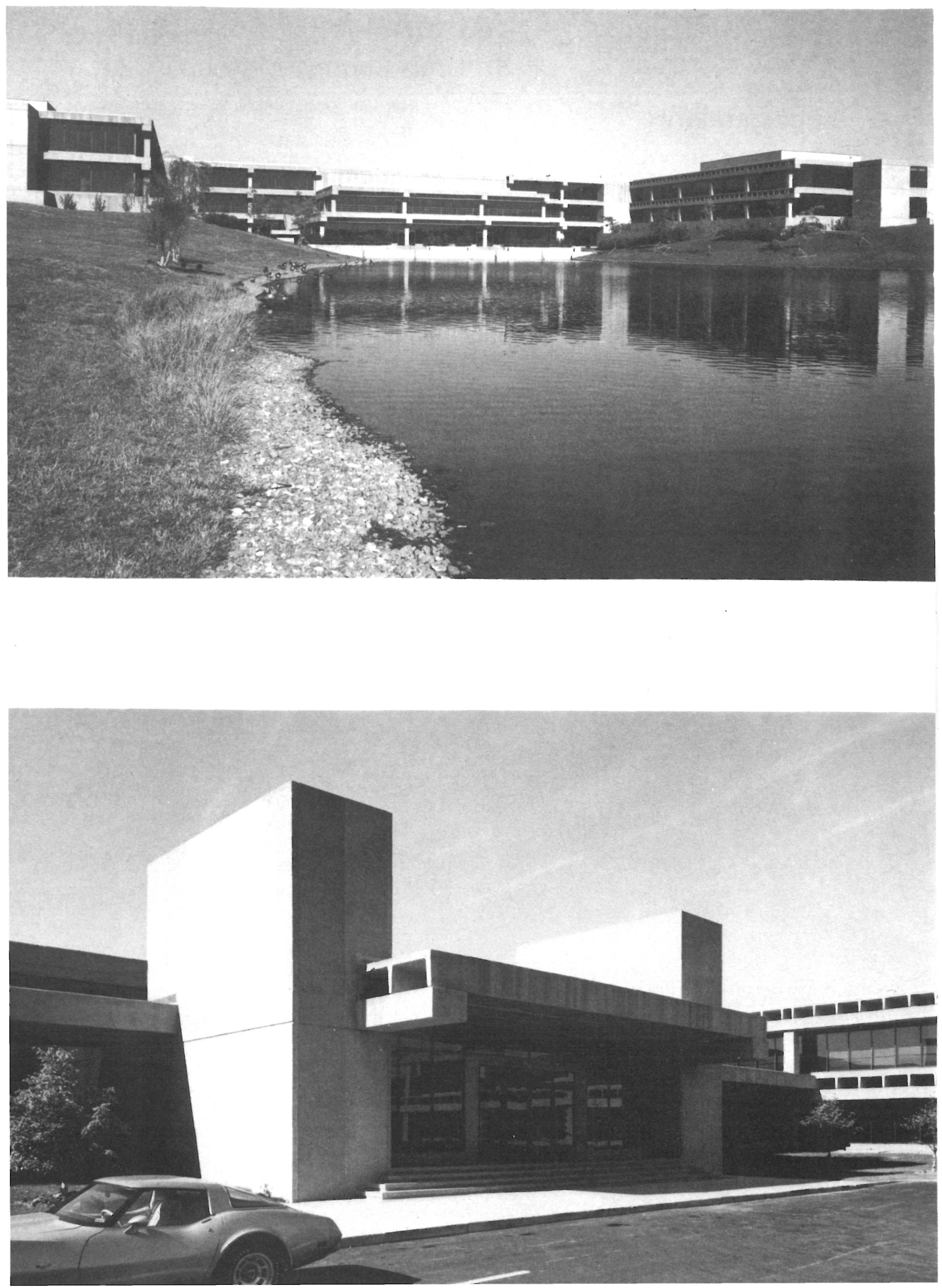

(c) Consejo Superior de Investigaciones Científicas Licencia Creative Commons 3.0 España (by-nc) 

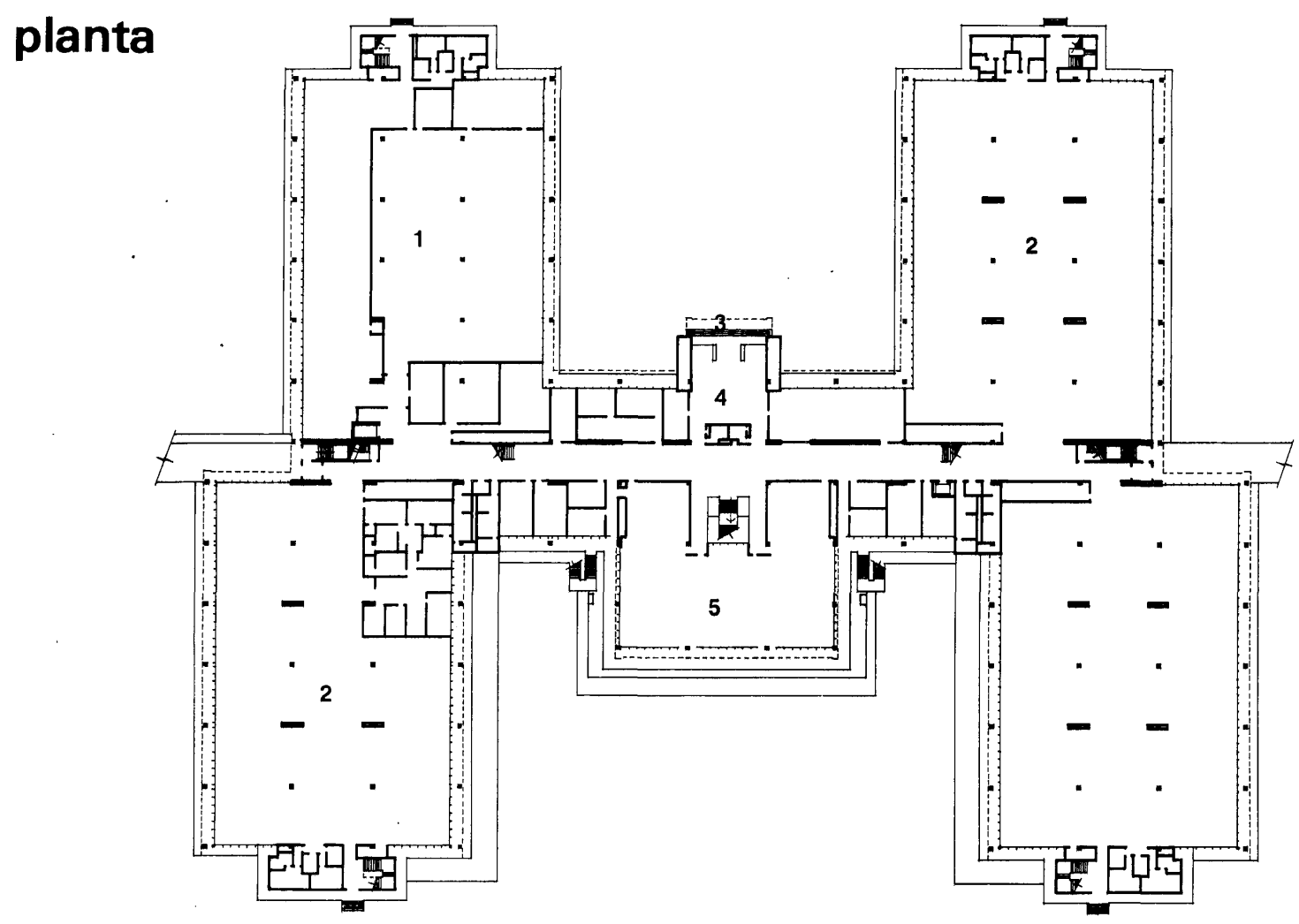

1. Centro de ordenadores; 2. Despachos; 3. Entrada; 4. Vestíbulo; 5 . Reuniones.

El National Service Center, situado en una parcela de más de $40 \mathrm{Ha}$ de superficie, distribuye un total de $23.000 \mathrm{~m}^{2}$ de oficinas y $7.500 \mathrm{~m}^{2}$ de almacenes y depósitos varios en un edificio bajo, de tres niveles de altura en su parte más alta. Un lago artificial, de hectárea y media de superficie, embellece los alrededores de la construcción, al tiempo que sirve para contener el agua de lluvia, dada la situación de la obra en un terreno de pendientes importantes.

El edificio adopta en planta la forma de una gran "H», de acuerdo a un eje de simetría, definiéndose cuatro cuerpos de construcción - dos a cada lado del eje-, articulados transversalmente mediante un corredor que conecta la escalera central con los núcleos de escaleras y ascensores situados en los extremos.

El primer nivel, situado a modo de semisótano, está dedicado fundamentalmente a contener depósitos y almacenes. El segundo nivel, o planta de acceso, contiene oficinas y áreas de recepción, predominando en su distribución el criterio de "espacio abierto", tanto para la organización interna de los despachos y zonas de trabajo como para la conexión de los espacios interiores con el contorno; esta disposición permite la entrada abundante de luz natural y el disfrute de los jardines desde las oficinas. El tercer y último nivel constituye una réplica del anterior en lo fundamental.

El proyecto procura la unidad entre la arquitectura y el entorno, y en este sentido alcanzan gran importancia los colores terrosos empleados en los acabados exteriores, escogidos por su compatibilidad con el ambiente rural. Muestra de ello son los grandes paneles de hormigón, prefabricados, que se emplearon en la construcción, con acabados de arena para darles el color y la textura adecuada, simulando la piedra clara. También la carpinteria exterior, que tiene color bronce oscuro como tono dominante, no sólo contribuye decisivamente a la definición de las fachadas, sino que proporciona una protección eficaz contra las inclemencias atmosféricas mediante el empleo de cristales térmicos de $2,5 \mathrm{~cm}$ de espesor. 

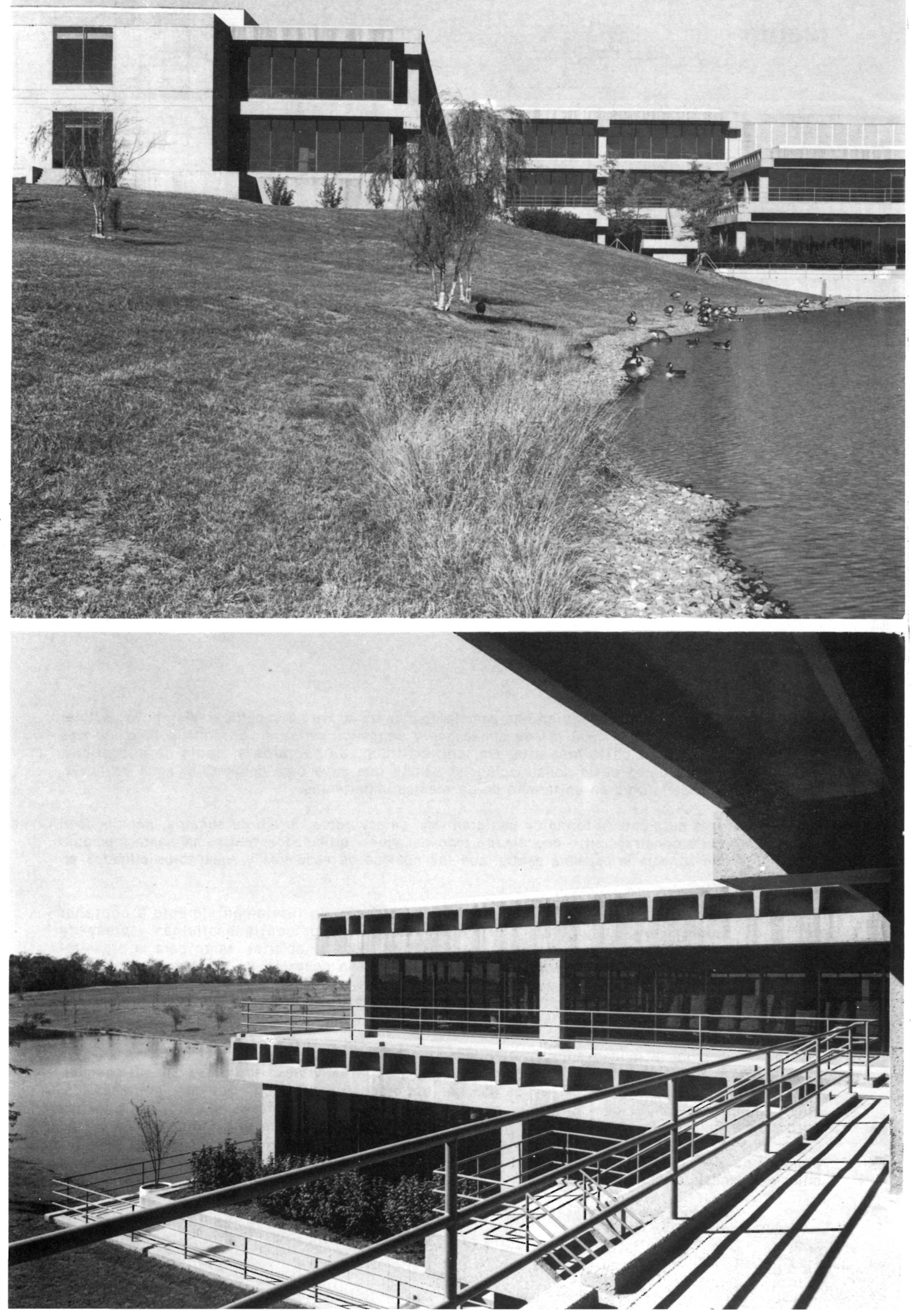

(C) Consejo Superior de Investigaciones Científicas Licencia Creative Commons 3.0 España (by-nc) 

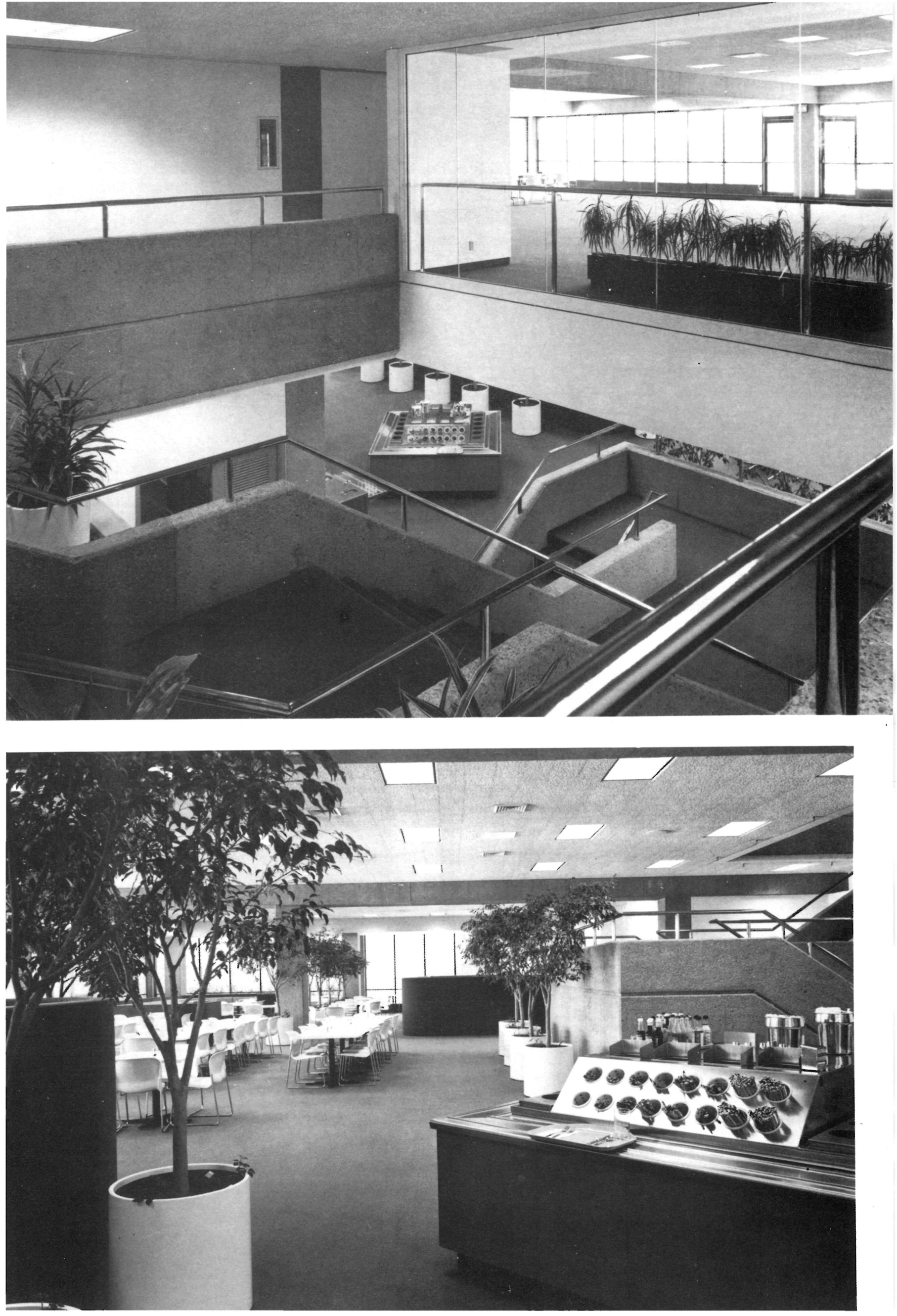

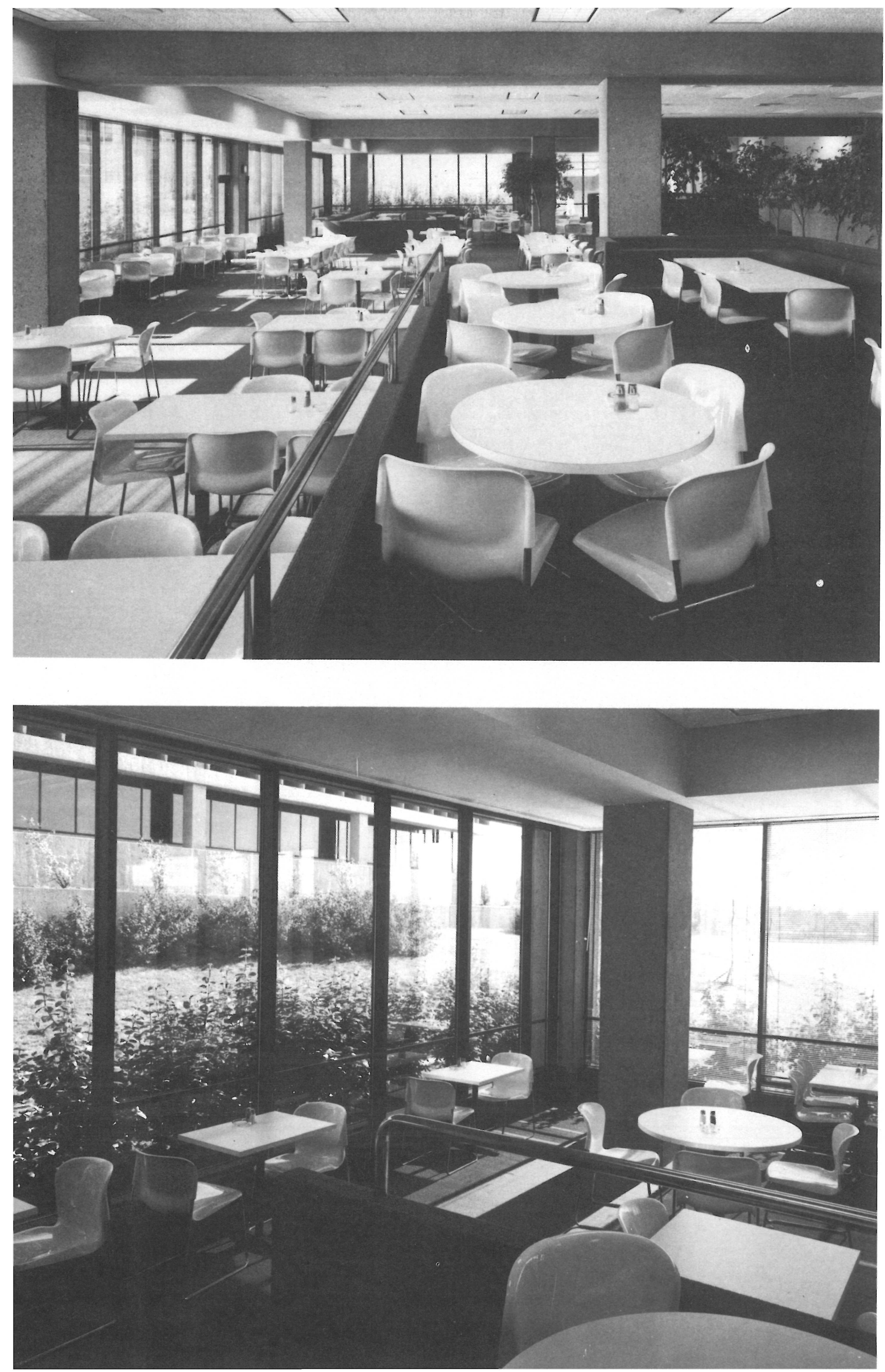
Exteriormente el complejo se caracteriza por la acusada horizontalidad de sus líneas arquitectónicas. En su interior se aprecia un alto grado de flexibilidad, con grandes espacios abiertos, libres de excesivas compartimentaciones que originen ambientes encajonados.

El empleo de módulos para la configuración de las plantas y de los volúmenes constituyen la clave del planteamiento arquitectónico, el cual permite una cómoda expansión de la construcción en el futuro. Se pueden añadir módulos enteros, o mitades de ellos, hasta duplicar la construcción existente en la actualidad, preservando siempre la unidad de diseño.

El capitulo de las instalaciones cobra gran importancia en esta obra, y asi se han aprovechado las vigas en "T», de hormigón, empleadas en la estructura horizontal, para alojar conductores cilindricos por donde circulan las canalizaciones de electricidad y aire acondicionado.

La energia eléctrica constituye la base de las instalaciones de calor y de aire frío. El complejo equipo de ordenadores empleado en las oficinas cuenta con un suministro continuo e ininterrumpido de energía, en previsión de fluctuaciones de la corriente normal. También destaca un sistema de seguridad basado en circuitos cerrados de televisión, con monitores situados en el hall principal de acceso.

FOTOS: KIKU OBATA Y PAULA CRANE
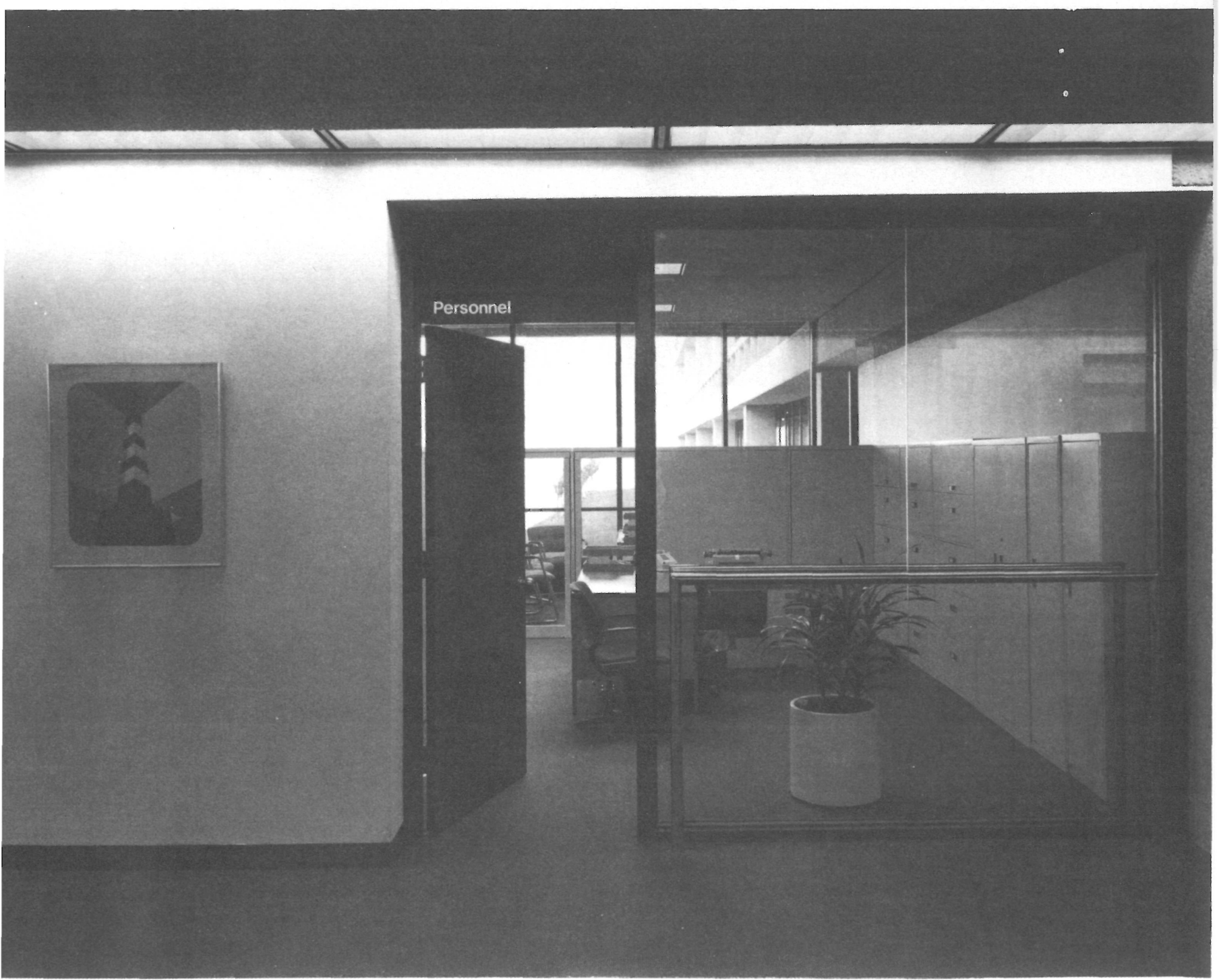


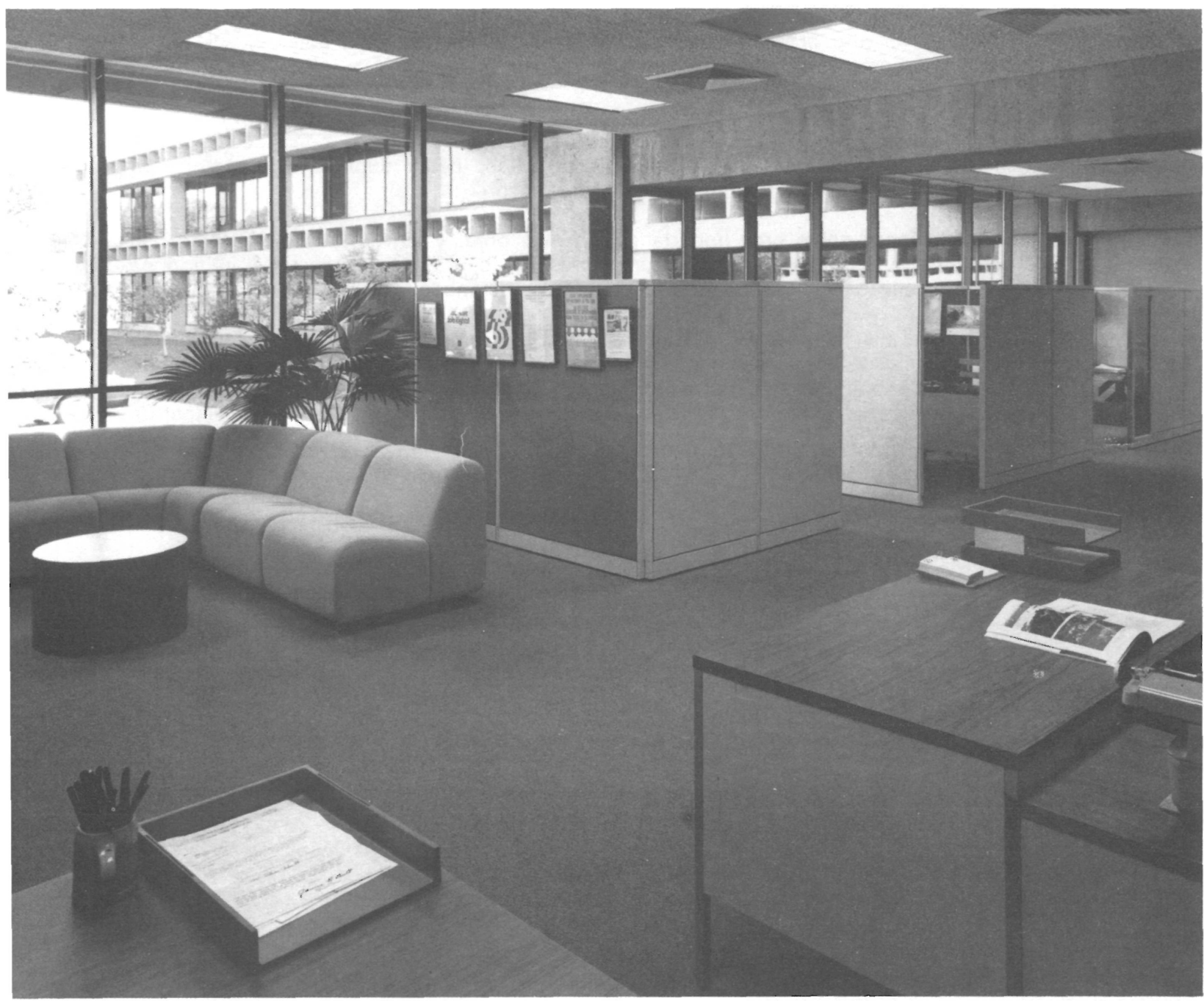

\section{résumé}

NATIONAL SERVICE CENTER,

St. Louis County, Missouri - USA

Hellmuth, Obata et Kassabaum,

architectes

Le nouveau siège du National Service Center, situé sur un terrain de plus de 40 ha dans la zone suburbaine, paraît un campus universitaire plutôt qu'un centre administratif. Le bâtiment, qui a seulement deux niveaux sur pente, fait partie d'un paysage nettement rural évitant la possible imposition agressive de l'architecture dans l'environnement. A cet effet, des couleurs terreuses ont été appliquées pour le finissage des panneaux préfabriqués de ciment, complé tés par une menuiserie d'une ligne nettement horizontale aux tons bronze foncé.

A l'intérieur, le bâtiment se distingue pour sa souplesse, formant des espaces de bureaux ouverts et intégrés. Sa structure modulaire permet l'agrandissement futur du bâtiment jusqu'à doubler ses dimensions, sans altérer l'unité architecturale.

\section{summary}

NATIONAL SERVICE CENTER, St. Louis County, Missouri - USA Hellmuth, Obata \& Kassabaum, Architects

The new building housing the National Service Center stands in a $40 \mathrm{Ha}$ property in
the outskirts of the city and it resembles more a university campus than an adminismore a university campus than an administrative centre. The building has only two levels above grade in an attempt to blend into the eminently rural surroundings, avoiding any architectural aggression to the natural environment. With a similar intention, earth hues were used in the precast concrete façade panels finish, completed by the horizontal emphasis of the dark bronze fenestration.

Interiorly, the building is outstanding by its flexible arrangement, creating open and integrated office spaces. The modular structure allows for future expansion that will eventually double its present size without affecting its architectural unity.

\section{zusammenfassung} NATIONAL SERVICE CENTER, St. Louis County, Missouri - USA

Hellmuth, Obata und Kassabaum, Architekten

Der neue Sitz des National Service Centers, der sich in einer Parzelle von über 40 ha am Aussenrand der Stadt befindet, gleicht eher einem Universitätskomplex als einem Verwaltungszentrum. Das nur zweistöckige Gebäude bemüht sich, eine Eingliederung in die stark ländliche Landschaft zu erzielen die stark landliche Landschaft zu erzielen und ein eventuelles agressives Beherrschen der Architektur innerhalb der natürlichen Umgebung zu vermeiden. Zu diesem Zweck fanden erdarbene Tonungen bel der Fertigstellung der vorgefertigten Zementplatten Anwendung. Die in ihrer Linienführung stark horizontalen Schreinereiarbeiten weisen dunkle Bronzefarben auf.

Im Innern fällt die Beweglichkeit des Gebäudes auf. Es wurden offene und einbezoene Büroräume geschaffen. Die moduläre Struktur ermöglicht eine zukünftige Erweiterung des Baus, welcher seine Abmessungen verdoppeln kann, ohne eine Veränderung de architektonischen Einheit hervorzurufen. 